\title{
On Fourth Order Centered Difference Scheme for Linear SPBVPs
}

\author{
A.M. Algelany ${ }^{1,3}$ E. R. El-Zahar ${ }^{1,2 *}$ \\ ${ }^{I}$ Department of Mathematics, College of Science and Humanities in Al-Kharj, \\ Prince Sattam bin Abdulaziz University, P.O. Box 83, Al-Kharj, 11942, Saudi Arabia. \\ ${ }^{2}$ Department of Basic Engineering Science, Faculty of Engineering, \\ Shebin El-Kom, 32511, Menofia University, Egypt \\ ${ }^{3}$ Department of Mathematics, Faculty of Sciences, Fayoum University, Fayoum, Egypt. \\ *Corresponding Author: ORCID: 0000-0001-7266-1893(E. R. El-Zahar)
}

\begin{abstract}
In this paper, a fourth order centered difference scheme is developed for linear second order singularly perturbed boundary value problems. The difference scheme is developed for the considered problem with variable coefficients and over unevenly spaced grid points. Uniqueness and stability condition is discussed at constant coefficients. Moreover, the convergence order is proved at evenly spaced grid.
\end{abstract}

Keywords: Finite difference centered techniques; unevenly spaced grid; convergence; stability.

\section{INTRODUCTION}

Singularly Perturbed Boundary Value Problems (SPBVPs) arise frequently in many fields of applied sciences particularly in the studies of fluid dynamics, quantum mechanics, chemical reactions, optimal control, etc. These problems have received a significant amount of attention in past and recent years [1-16].

It is well known that away from the boundary layers standard upwind difference schemes over uniform mesh can be used and accurate results are obtained. Otherwise, other integration schemes are to be preferred such as difference schemes on a non-uniform mesh, variable step-size schemes, etc [1-6, 8-15]. The main difficulty in global discretization of SPBVPs is the restriction on the step- size that to have a unique stable and accurate solution. Therefore stability and order of convergence act as the major achieved requirements. Many authors deal with some of these challenges in global discretization for these problems especially the convection diffusion problems [2-6]. Segal [2] analyzed and compared various methods for solving the convection diffusion equation with small perturbation parameter. While Il'in's [3] method is a very accurate example of an upwind scheme for a homogeneous, one-dimensional convection-diffusion equation with constant coefficients. It loses accuracy when variable coefficients are used. Dekema and Schultz [4] developed high-order methods to solve elliptic problems and obtained remarkably good numerical results. Later, Choo and Schultz
[5] developed the so-called stable central difference methods. They modified the central difference approximations for the first- and second-order derivatives by rewriting its error terms as a combination of the lower-order derivative terms and approximating them. This process reinforced the diagonal dominance of the coefficient matrix and had a stabilizing effect. However, they could not achieve as high accuracy as the method of Dekema and Schultz [4]. Ilicasu and Schultz [6] developed high-order methods to solve singular perturbation problems. They rewrote higher order derivatives in Taylor expansion in terms of the lower-order derivative terms. However, they also used constant coefficients only. Most the above techniques go away from using non-uniform grid points. The main reason is the complexity of driving general formulas that will solve these problems. Moreover, this leads to more complicated studying of uniqueness, stability, and convergence. Now, using mathematical symbolic languages such as Maple, Drive and Mathematica make the mission easier than earlier. In this paper, following the idea in $[5,6,8$, 16] a fourth order centered difference scheme is developed for linear second order singularly perturbed boundary value problems. The difference scheme is developed for the considered problem with variable coefficients and over unevenly spaced grid points. Uniqueness and stability condition is discussed at constant coefficients. Moreover, the convergence order is proved at evenly spaced grid.

\section{FOURTH FINITE DIFFERENCE SCHEME}

Consider the following linear SPBVP

$$
L(y) \equiv-\varepsilon y^{\prime \prime}+p(x) y^{\prime}+q(x) y=f(x), \quad a \leq x \leq b,
$$

with boundary conditions

$$
y(a)=\alpha \text { and } y(b)=\beta
$$

where $\varepsilon$ is a small positive parameter $(0<\varepsilon<<1), \alpha$ and $\beta$ are given constants, $p(x), q(x)$ and $f(x)$ are assumed to be sufficiently continuously differentiable functions on $[a, b]$, 
International Journal of Engineering Research and Technology. ISSN 0974-3154, Volume 13, Number 2 (2020), pp. $364-367$

(C) International Research Publication House. https://dx.doi.org/10.37624/IJERT/13.2.2020.364-367

Moreover assume $q(x)>0, p(x)<P<0$ for all $x \in[a, b]$, where $P$ is some negative constant. Under these assumptions, SPBVP (1) has a unique solution which in general displays a boundary layer of width $O(\varepsilon)$ at $x=a[2,4-16]$. First, $[a, b]$ is divided into $N$ subintervals such that $x_{0}=a<x_{1}<x_{2}<\ldots \ldots<x_{N}=b$ with step size $h_{i}=x_{i}-x_{i-1}$, $i=1,2, \ldots . . N$. For the sake of simplicity, we use $p_{i}=p\left(x_{i}\right)$, $q_{i}=q\left(x_{i}\right), f_{i}=f\left(x_{i}\right), y_{i-1}=y\left(x_{i-1}\right), \quad y_{i+1}=y\left(x_{i+1}\right), \quad$ and $y_{i}^{\prime}=y^{\prime}\left(x_{i}\right)$, etc. The solution of SPBVP (1) is approximated over subintervals with three grid points as shown in figure 1.

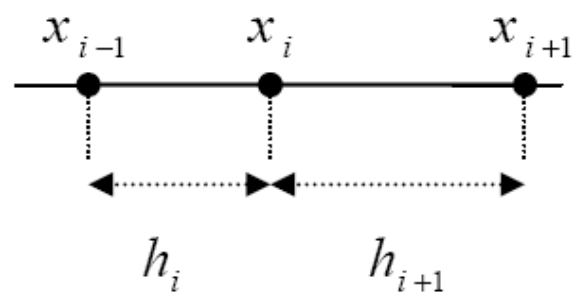

Figure 1. Unevenly spaces grid points over subintervals

At each point $x_{i}$ we want to find $E_{i}, F_{i}, G_{i}, H_{i}$ such that

$$
y_{i}^{\prime \prime}-\omega p_{i} y_{i}^{\prime}-\omega q_{i} y_{i}=E_{i} y_{i-1}+F_{i} y_{i}+G_{i} y_{i+1}+H_{i}=-\omega f_{i}
$$

where $\omega=1 / \varepsilon$.These terms are obtained using Taylor series expansions of $y_{i+1}$ and $y_{i-1}$ around $x_{i}$ in (2) as follows

$$
\begin{array}{r}
y_{i}^{\prime \prime}-\omega p_{i} y_{i}^{\prime}-\omega q_{i} y_{i}=F_{i} y_{i}+H_{i}+G_{i}\left[y_{i}+h_{i} y_{i}^{\prime}+\frac{h_{i+1}^{2}}{2} y_{i}^{\prime \prime}\right. \\
\left.+\frac{h_{i+1}^{3}}{6} y_{i}^{\prime \prime \prime}+. .\right]+E_{i}\left[y_{i}-h_{i} y_{i}^{\prime}+\frac{h_{i}^{2}}{2} y_{i}^{\prime \prime}-\frac{h_{i}^{3}}{6} y_{i}^{\prime \prime \prime}+. .\right]
\end{array}
$$

From Eq. (1) we have

$$
\begin{aligned}
y_{i}^{\prime \prime \prime}= & \omega p_{i} y_{i}^{\prime \prime}+\omega\left(p_{i}^{\prime}+q_{i}\right) y_{i}^{\prime}+\omega q_{i}^{\prime} y_{i}-\omega f_{i}^{\prime}, \\
y_{i}^{(4)}=[ & {\left[\omega^{2} p_{i}^{2}+2 \omega p_{i}^{\prime}+\omega q_{i}\right] y_{i}^{\prime \prime}-\omega f_{i}^{\prime \prime}+\left[\omega^{2} p_{i}\left(p_{i}^{\prime}+q_{i}\right)+\right.} \\
+ & \left.\omega\left(p_{i}^{\prime \prime}+2 q_{i}^{\prime}\right)\right] y_{i}^{\prime}+\left[\omega^{2} p_{i} q_{i}^{\prime}+\omega q_{i}^{\prime \prime}\right] y_{i}-\omega^{2} p_{i} f_{i}^{\prime} \\
y_{i}^{(5)}= & {\left[\omega^{3} p_{i}^{3}+5 \omega^{2} p_{i}^{\prime} p_{i}+3 \omega p_{i}^{\prime \prime}+3 \omega q_{i}^{\prime}+2 \omega^{2} p_{i} q_{i}\right] y_{i}^{\prime \prime} } \\
& +\left[\omega^{3} p_{i}^{2} p_{i}^{\prime}+\omega^{3} p_{i}^{2} q_{i}+3 \omega^{2} p_{i}^{\prime 2}+4 \omega^{2} p_{i}^{\prime} q_{i}+3 \omega q_{i}^{\prime \prime}+\right. \\
& \left.+2 \omega^{2} p_{i} q_{i}^{\prime}+\omega^{2} p_{i} p_{i}^{\prime \prime}+\omega^{2} q_{i}^{2}+\omega p_{i}^{\prime \prime \prime}\right] y_{i}^{\prime}+\left[\omega^{3} p_{i}^{2} q_{i}^{\prime}+\right. \\
& \left.+3 \omega^{2} p_{i}^{\prime} q_{i}^{\prime}+\omega^{2} q_{i} q_{i}^{\prime}+\omega^{2} p_{i} q_{i}^{\prime \prime}+\omega q_{i}^{\prime \prime \prime}\right] y_{i}- \\
& -\left[\omega^{3} p_{i}^{2} f_{i}^{\prime}+3 \omega^{2} p_{i}^{\prime} f_{i}^{\prime}+\omega^{2} q_{i} f_{i}^{\prime}+\omega^{2} p_{i} f_{i}^{\prime \prime}+\omega f_{i}^{\prime \prime \prime}\right] .
\end{aligned}
$$

Substituting Eqs. (4)-(6) in Eq.(3) and equating the coefficients of $y_{i}, y_{i}^{\prime}$ and $y_{i}^{\prime \prime}$, taking the sixth derivative terms are the largest contributors to the error, results in

$$
\begin{aligned}
E_{i}= & 24\left(h_{i+1}^{4} \omega\left(p_{i}^{4} \omega^{3}+3 p_{i}^{2} \omega^{2}\left(2 p_{i}^{\prime}+q_{i}\right)+\omega \theta_{1}+p_{i}^{\prime \prime \prime}+3 q_{i}^{\prime \prime}\right),\right. \\
& \left.+5 h_{i+1}^{3} \omega \theta_{2}+20 h_{i+1}^{2} \omega \theta_{3}+60 h_{i+1} p_{i} \omega+120\right) / h_{i} / T \\
G_{i}= & 24\left(h_{i}^{4} \omega\left(p_{i}^{4} \omega^{3}+3 p_{i}^{2} \omega^{2}\left(2 p_{i}^{\prime}+q_{i}\right)+\omega \theta_{1}+p_{i}^{\prime \prime \prime}+3 q_{i}^{\prime \prime}\right),\right. \\
& \left.-5 h_{i}^{3} \omega \theta_{2}+20 h_{i}^{2} \omega \theta_{3}-60 h_{i} p \omega+120\right) / h_{i+1} / T \\
F_{i}= & G_{i}\left[-1-\frac{h_{i+1}^{4} \omega\left(q_{i}^{\prime} p_{i} \omega+q_{i}^{\prime \prime}\right)}{24}-\frac{h_{i+1}^{3} q_{i}^{\prime} \omega}{6}-\frac{\omega h_{i+1}^{5}}{120} \theta_{4}\right], \\
+ & E_{i}\left[-1-\frac{h_{i}^{4} \omega\left(q_{i}^{\prime} p_{i} \omega+q_{i}^{\prime \prime}\right)}{24}+\frac{h_{i}^{3} q_{i}^{\prime} \omega}{6}+\frac{\omega h_{i}^{5}}{120} \theta_{4}\right]-\omega q_{i} \\
H_{i}= & -G_{i}\left[-\frac{h_{i+1}^{4} \omega\left(f_{i}^{\prime} p_{i} w+f_{i}^{\prime \prime}\right)}{24}-\frac{h_{i+1}^{3} f_{i}^{\prime} \omega}{6}-\frac{\omega h_{i+1}^{3}}{120} \theta_{5}\right], \\
- & E_{i}\left[-\frac{h_{1}^{4} \omega\left(f_{i}^{\prime} p_{i} \omega+f_{i}^{\prime \prime}\right)}{24}+\frac{h_{1}^{3} f_{i}^{\prime} \omega}{6}+\frac{\omega h_{1}^{5}}{120} \theta_{5}\right]
\end{aligned}
$$

where

$$
\begin{aligned}
& \theta_{1}=\left(3 p_{i}^{\prime 2}+4 p_{i}^{\prime} q_{i}+4 p_{i}^{\prime \prime} p_{i}+5 q_{i}^{\prime} p_{i}+5 q_{i}^{\prime} p_{i}+q_{i}^{2}\right) \\
& \theta_{2}=\left(p_{i}^{3} \omega^{2}+p \omega\left(3 p_{i}^{\prime}+2 q_{i}\right)+p_{i}^{\prime \prime}+2 q_{i}^{\prime}\right) \\
& \theta_{3}=\left(p_{i}^{2} \omega+p_{i}^{\prime}+q_{i}\right) \\
& \theta_{4}=\left(\omega q_{i}^{\prime}\left(\omega p_{i}^{2}+3 p_{i}^{\prime}+q_{i}\right)+\omega p_{i} q_{i}^{\prime \prime}+q_{i}^{\prime \prime \prime}\right) \\
& \theta_{5}=\left(\omega f_{i}^{\prime}\left(\omega p_{i}^{2}+3 p_{i}^{\prime}+q_{i}\right)+\omega p_{i} f_{i}^{\prime \prime}+f_{i}^{\prime \prime}\right) \\
& T=h_{i}^{4} \omega\left(\omega^{2}\left(h_{i+1}^{3} k_{1}-4 h_{i+1} p_{i}\left[k_{2}-3 p_{i} k_{5}\right]-24 p_{i}^{3}\right)+\omega\left(h_{i+1}^{3}\right.\right. \\
& \left(2 p_{i}^{\prime} k_{6}-3 p_{i}^{\prime \prime 2}-9 p_{i}^{\prime \prime} q_{i}^{\prime}+p_{i}^{\prime \prime \prime} q_{i}-3\left(2 q_{i}^{\prime 2}-q_{i}^{\prime \prime} q_{i}\right)\right)-4 h_{i+1}^{2} k_{3}+ \\
& \left.\left.12 h_{i+1} k_{4}-24\left(5 p_{i}^{\prime}+2 q_{i}\right)\right)+12\left(h_{i+1} k_{6}-6\left(p_{i}^{\prime \prime}+q_{i}^{\prime}\right)\right)\right)+h_{i}^{3} \omega \\
& \left(h_{i+1}^{4} \omega^{2} k_{1}+\omega\left(h _ { i + 1 } ^ { 4 } \left(2 p_{i}^{\prime} k_{6}-3 p_{i}^{\prime \prime 2}-9 p_{i}^{\prime \prime} q_{i}^{\prime}+p_{i}^{\prime \prime \prime} q_{i}-3\left(2 q_{i}^{\prime 2}\right.\right.\right.\right. \\
& \left.\left.\left.-q_{i}^{\prime \prime} q_{i}\right)\right)+20 h_{i+1}^{2} k_{2}-60 h_{i+1} p_{i} k_{5}+120 p_{i}^{2}\right)-60\left(h _ { i + 1 } \left(p_{i}^{\prime \prime}+\right.\right. \\
& \left.\left.\left.+2 q_{i}^{\prime}\right)-2\left(2 p_{i}^{\prime}+q_{i}\right)\right)\right)+4 h_{i}^{2} \omega\left(h_{i+1}^{4} p_{i} \omega^{2} k_{2}+h_{i+1}^{3} \omega\left(h_{i+1} k 3\right.\right. \\
& \left.\left.+5 k_{2}\right)+60\left(h_{i+1} k_{5}-2 p_{i}\right)\right)+12 h_{i+1}\left(h_{i+1}^{4} p_{i}{ }^{2} \omega^{3} k_{5}+h_{i+1}^{3} \omega^{2}\right. \\
& \left(h_{i+1} k_{4}++5 p_{i} k_{5}\right)+h_{i+1}^{2} \omega\left(h_{i+1}^{2} k_{6}+5 h_{i+1}\left(p_{i}^{\prime \prime \prime}+2 q_{i}^{\prime}\right)+20 k_{5}\right) \\
& +120)+24 h_{i+1}\left(h_{i+1}^{3} p_{i}{ }^{3} \omega^{3}+h_{i+1}^{2} p_{i} \omega^{2}\left(h_{i+1}\left(5 p_{i}^{\prime}+2 q_{i}\right)+5 p_{i}\right)+\right. \\
& \left.+h_{i+1} \omega\left(3 h_{i+1}^{2}\left(p_{i}^{\prime \prime}+q_{i}^{\prime}\right)+5 h_{i+1}\left(2 p_{i}^{\prime}+q_{i}\right)+20 p_{i}\right)+60\right) \\
& \text { and } \\
& k_{1}=\left(6 p_{i}^{\prime 3}+11 p_{i}^{\prime 2} q-3 p^{\prime}\left(2 p_{i}^{\prime \prime} p_{i}+3 q_{i}^{\prime} p_{i}-2 q_{i}{ }^{2}\right)-4 p_{i}^{\prime \prime} p_{i} q_{i}\right. \\
& \left.+p_{i}^{\prime \prime} p_{i}{ }^{2}-5 q_{i}^{\prime} p_{i} q_{i}+3 q_{i}^{\prime \prime} p_{i}{ }^{2}+q_{i}{ }^{3}\right) \\
& k_{2}=\left(2 p_{i}^{\prime 2}+3 p_{i}^{\prime} q_{i}-p_{i}^{\prime \prime} p_{i}-2 q_{i}^{\prime} p_{i}+q_{i}^{2}\right) \\
& k_{3}=\left(3 p_{i}^{\prime}\left(p_{i}^{\prime \prime}+q_{i}^{\prime}\right)+3 p_{i}^{\prime \prime} q_{i}-p_{i}^{\prime \prime \prime} p_{i}+3 q_{i}^{\prime} q_{i}-3 q_{i}^{\prime \prime} p_{i}\right) \\
& k_{4}=\left(3 p_{i}^{\prime 2}+4 p_{i}^{\prime} q_{i}+p_{i}^{\prime \prime} p_{i}+2 q_{i}^{\prime} p_{i}+q_{i}{ }^{2}\right) \\
& k_{5}=\left(p_{i}^{\prime}+q_{i}\right), \quad k_{6}=\left(p_{i}^{\prime \prime \prime}+3 q_{i}^{\prime \prime}\right) \text {. }
\end{aligned}
$$


Then the difference equation and the local truncation error $\tau_{i}$ are introduced as:

$$
\begin{aligned}
& E_{i} y_{i-1}+F_{i} y_{i}+G_{i} y_{i+1}=-H_{i}-w f_{i}+\tau_{i}, \\
& \tau_{i}=\left(G_{i} \frac{h_{i+1}^{6}}{720} y^{(6)}(\xi)+E_{i} \frac{h_{i}^{6}}{720} y^{(6)}(\zeta)\right),
\end{aligned}
$$

where $\xi \in\left[x_{i}, x_{i+1}\right], \zeta \in\left[x_{i-1}, x_{i}\right]$.

\section{UNIQUENESS AND STABILITY CONDITION}

The existence and uniqueness of the solution for the present difference scheme is shown by establishing that the tridiagonal coefficient matrix of the result algebraic system is diagonally dominant with negative main diagonal elements and positive super-diagonal and sub-diagonal elements.

The nominators of $E_{i}$ and $G_{i}$, in Eqs. (7)- (8) at constant coefficients $p=p(x)$ and $q=q(x)$, are positive when

$$
\begin{aligned}
& 24\left(h_{i+1}^{4}\left(p^{4} \omega^{4}+3 p^{2} \omega^{3} q+\omega^{2} q^{2}\right)+5 h_{i+1}^{3}\left(p^{3} \omega^{3}+\right.\right. \\
& \left.\left.\quad+2 \omega^{2} p q\right)+20 h_{i+1}^{2} \omega\left(p^{2} \omega+q\right)+60 h_{i+1} p \omega+120\right)>0
\end{aligned}
$$

and

$$
\begin{aligned}
& 24\left(h_{i}^{4}\left(p^{4} \omega^{4}+3 p^{2} \omega^{3} q+\omega^{2} q^{2}\right)-5 h_{i}^{3}\left(p^{3} \omega^{3}+\right.\right. \\
& \left.\left.\quad+2 \omega^{2} p q\right)+20 h_{i}^{2} \omega\left(p^{2} \omega+q\right)-60 h_{i} p \omega+120\right)>0
\end{aligned}
$$

which are equivalent to

$$
\begin{aligned}
& 24\left(h_{i}^{4}\left(p^{4} \omega^{4}+3 p^{2} \omega^{3} q+\omega^{2} q^{2}\right)-5 h_{i}^{3}\left(|p|^{3} \omega^{3}+\right.\right. \\
& \left.\left.\quad+2 \omega^{2}|p| q\right)+20 h_{i}^{2} \omega\left(p^{2} \omega+q\right)-60 h_{i}|p| \omega+120\right)>0
\end{aligned}
$$

The denominator of $E_{i}$ and $G_{i}$, in Eqs.(7)-(8) at constant coefficients $p=p(x)$ and $q=q(x)$, are positive when

$$
\begin{aligned}
& {\left[1440+\left(\omega q h_{i} h_{i+1}\right)^{3}+20\left(\omega q h_{i} h_{i+1}\right)^{2}+240\left(\omega q h_{i} h_{i+1}\right)\right]+} \\
& +4 \omega p\left(h_{i+1}-h_{i}\right)\left[120+15\left(\omega q h_{i} h_{i+1}\right)+\left(\omega q h_{i} h_{i+1}\right)^{2}\right]>0
\end{aligned},
$$

and

$$
\begin{aligned}
12 \omega\left(h_{i+1}^{3}\right. & \left.+h_{i}^{3}\right)\left(\omega p^{2}+q\right)\left[10+\omega q h_{i} h_{i+1}\right]+ \\
& +\left(h_{i+1}^{4}-h_{i}^{4}\right)\left[24 \omega^{2} p\left(p^{2} \omega+2 q\right)\right]>0
\end{aligned}
$$

After simplification and manipulation using Mathematica, solving these inequalities results in

$$
h_{i+1}, h_{i+1}<\min \left(\frac{2}{\omega|p|}, \frac{2|p|}{q}\right)
$$

Thus the present difference scheme is stable and has a unique solution under the condition (18).

\section{ORDER OF CONVERGENCE}

Assume that the present scheme is equivalent to those in approximating the second and the first derivatives in (1) using Taylor's expansion at fixed step size $h$ as follows:

$$
y^{\prime \prime}\left(x_{i}\right)=y_{i}^{\prime \prime}+O\left(h^{k}\right), \quad y^{\prime}\left(x_{i}\right)=y_{i}^{\prime}+O\left(h^{k}\right)
$$

Then the order of convergence of the present scheme can be determined from equations (11), (12) and (19) as follows.

$$
y_{i}^{\prime \prime}+O\left(h^{k}\right)-w p_{i}\left(y_{i}^{\prime}+O\left(h^{k}\right)\right)-w q_{i} y_{i}=w f_{i}+\tau_{i}
$$

and

$$
O\left(h^{k}\right)=\frac{\tau_{i}}{1-\omega},
$$

Using (12) and (21), the local truncation as $\varepsilon \rightarrow 0$ is given by

$$
\left.\tau\right|_{w \rightarrow \infty}=\left(\frac{p^{4}}{q\left(h^{2} q^{2}+12 p^{2}\right)}\right)\left(\frac{h^{4}}{30}\right) y^{(6)}(\zeta),
$$

Thus the present difference scheme has a fourth order of convergence.

\section{CONCLUSION AND DISCUSSION}

In this paper, we have developed a fourth order centered difference scheme for solving linear second order singularly perturbed boundary value problems. The difference scheme is developed for the SPBVP with variable coefficients over unevenly spaced grid points. The uniqueness and stability condition is discussed at constant coefficients. The order of convergence is proved at evenly spaced grid. The paper draws the attention of researchers to drive general formulas and perform deeply more complicated studying and analysis of uniqueness, stability and convergence for SPBVPs with variable coefficients over unevenly spaced grid points aided mathematical symbolic languages.

\section{ACKNOWLEDGEMENT.}

The authors acknowledge that this publication was supported by the Deanship of Scientific Research at Prince Sattam bin Abdulaziz University.

\section{REFERENCES}

[1] Ascher, U Mattheij, RMM. Russel, R.D. Numerical Solution of Boundary Value Problems for ordinary Differential Equations (Prentice-Hall, Englewood Cliffs, N J, 1988).

[2] Segal, A. Aspects of numerical methods for elliptic singular perturbation problems, SIAM J. Sci. Stat.Comput. 3(1982) 327-349. 
[3] Dekema, SK., Schultz, DH. High-order methods for differential equations with large first-derivative terms, Int. J. Num. Methods Fluids 10, 259-284, (1990).

[4] Choo, JY. Schultz, DH. Stable high order methods for differential equations with small coefficients for the second order terms, Computers Math. Applic, 25 (1993)105-123.

[5] Il'in, AM. Differencing scheme for a differential equation with a small parameter affecting the highest derivative, Math. Notes Acad. SC. USSR 6, (1969) 596-602.

[6] ILICASU, F0. SCHULTZ, D.H. High-Order FiniteDifference Techniques for Linear Singular Perturbation Boundary Value Problems, Computers Math. Applic, 47 (2004) 391-417

[7] Vulanovic,R. Novi Sad. A uniform numerical method for quasilinear singular perturbation problems without turning points, Computing 41, 97-106(1989).

[8] Alessandra Papini, About the central difference method for singularly perturbed boundary value problems, Appl. Num. Math, 17 (1995) 333-346

[9] Weiss, R. An analysis of the box and trapezoidal schemes for linear singularly perturbed boundary value problems, Math. Comp, 42 (1984)41-67.

[10] Varner, TN. and Choudhury, S.R. Nonstandard difference schemes for singular perturbation problems revisited, Appl. Math. Comp, 92, 101-123, (1998).

[11] Habib HM, El-Zahar ER. An algorithm for solving singular perturbation problems with mechanization. Appl Math Comput, 2007, 188:286-302.

[12] El-Zahar, ER. Piecewise approximate analytical solutions of high order singular perturbation problems with a discontinuous source term, International Journal of Differential Equations, vol. 2016, Article ID 1015634, 12 pages, 2016. doi:10.1155/2016/1015634

[13] El-Zahar, ER. Applications of Adaptive Multi step Differential Transform Method to Singular Perturbation Problems Arising in Science and Engineering, Applied Mathematics and Information Sciences, 2015, 9(1), 223-232.

[14] El-Zahar, ER. Approximate analytical solutions of singularly perturbed fourth order boundary value problems using differential transform method, Journal of King Saud University (Science), 2013, 25(3), 257265.

[15] El-Zahar, ER, EL-Kabeir, SM. A new method for solving singularly perturbed boundary value problems, Applied Mathematics and Information Sciences., 2013, 7(3), 927-938.

[16] El-Zahar, ER. Algelany AM. On Three-Point Finite Difference Techniques for SPBVPs, International Journal of Engineering Research and Technology, 2019, 13(2), 229-232. 